\title{
Non-Compact and Sharp Embeddings of Logarithmic Bessel Potential Spaces into Hölder-Type Spaces
}

\author{
David E. Edmunds, Petr Gurka and Bohumír Opic
}

\begin{abstract}
In our recent paper [Compact and continuous embeddings of logarithmic Bessel potential spaces. Studia Math. 168 (2005), 229 - 250] we have proved an embedding of a logarithmic Bessel potential space with order of smoothness $\sigma$ less than one into a space of $\lambda(\cdot)$-Hölder-continuous functions. We show that such an embedding is not compact and that it is sharp.
\end{abstract}

Keywords. Generalized Lorentz-Zygmund spaces, logarithmic Bessel potential spaces, Hölder-continuous functions, embeddings

Mathematics Subject Classification (2000). Primary 46E35, secondary 46E30, $26 \mathrm{D} 15$

\section{Introduction}

In the recent paper [8] we have derived embeddings of Bessel potential spaces with smoothness $\sigma \in(0,1)$, modelled upon generalized Lorentz-Zygmund spaces, into spaces of $\lambda(\cdot)$-Hölder-continuous functions. Here we discuss non-compactness and sharpness of those embeddings.

To be more specific, we need some notation. Given two (quasi-)Banach spaces $X$ and $Y$, we write $X \hookrightarrow Y$ or $X \hookrightarrow \hookrightarrow Y$ if $X \subset Y$ and the natural embedding is continuous or compact, respectively.

Let $p, q \in(0, \infty], m \in \mathbb{N}, \alpha_{1}, \ldots, \alpha_{m} \in \mathbb{R}$ and let $\Omega$ be a measurable subset of $\mathbb{R}^{n}$ (with respect to $n$-dimensional Lebesgue measure). The generalized

D. E. Edmunds: School of Mathematics, Cardiff University, Senghennydd Road, Cardiff CF24 4YH; davideedmunds@aol.com

P. Gurka: Department of Mathematics, Czech University of Agriculture, 16521 Prague 6, Czech Republic; gurka@tf.czu.cz

B. Opic: Mathematical Institute, Academy of Sciences of Czech Republic, Žitná 25, 11567 Prague 1, Czech Republic; opic@math.cas.cz

The research was partially supported by grant no. 201/01/0333 of the Grant Agency of the Czech Republic, by NATO grant PST. CLG. 978694, and by Leverhulme Trust grant $\mathrm{F} / 00407 / \mathrm{E}$. 
Lorentz-Zygmund (GLZ) space $L_{p, q ; \alpha_{1}, \ldots, \alpha_{m}}(\Omega)$ consists of all measurable (real or complex) functions $f$ on $\Omega$ such that the quantity

$$
\|f\|_{p, q ; \alpha_{1}, \ldots, \alpha_{m}}:=\left\|t^{\frac{1}{p}-\frac{1}{q}}\left(\prod_{j=1}^{m} \ell_{j}^{\alpha_{j}}(t)\right) f^{*}(t)\right\|_{q,(0, \infty)}
$$

is finite. Here $\ell_{1}, \ldots, \ell_{m}$ are (logarithmic) functions defined on $(0, \infty)$ by

$$
\ell_{1}(t)=\ell(t)=1+|\log t|, \quad \ell_{j}(t)=1+\log \ell_{j-1}(t) \quad(j>1),
$$

$f^{*}$ denotes the non-increasing rearrangement of $f$ given by

$$
f^{*}(t)=\inf \left\{\lambda>0 ;|\{x \in \Omega ;|f(x)|>\lambda\}|_{n} \leq t\right\}, \quad t \geq 0,
$$

$|G|_{n}$ stands for the $n$-volume of a measurable subset $G$ of $\mathbb{R}^{n}$ and $\|\cdot\|_{q,(a, b)}$ is the usual $L^{q}$-(quasi-)norm on an interval $(a, b) \subseteq \mathbb{R}$. (For more details about the spaces $L_{p, q ; \alpha_{1}, \ldots, \alpha_{m}}(\Omega)$ see [2]-[7], [9], and [11].)

The Bessel kernel $g_{\sigma}, \sigma>0$, is defined to be that function on $\mathbb{R}^{n}$ whose Fourier transform $\widehat{g}_{\sigma}$ is

$$
\widehat{g}_{\sigma}(\xi)=(2 \pi)^{-\frac{n}{2}}\left(1+|\xi|^{2}\right)^{-\frac{\sigma}{2}}, \quad \xi \in \mathbb{R}^{n},
$$

where by the Fourier transform $\widehat{f}$ of a function $f$ we mean

$$
\widehat{f}(x)=(2 \pi)^{-\frac{n}{2}} \int_{\mathbb{R}^{n}} e^{-i x y} f(y) \mathrm{d} y, \quad x \in \mathbb{R}^{n} .
$$

Let $\sigma>0, p \in(1, \infty), q \in[1, \infty], \alpha_{1}, \ldots, \alpha_{m} \in \mathbb{R}$. The logarithmic Bessel potential space $H^{\sigma} L_{p, q ; \alpha_{1}, \ldots, \alpha_{m}}\left(\mathbb{R}^{n}\right)$ is defined by

$$
H^{\sigma} L_{p, q ; \alpha_{1}, \ldots, \alpha_{m}}\left(\mathbb{R}^{n}\right):=\left\{u=g_{\sigma} * f ; f \in L_{p, q ; \alpha_{1}, \ldots, \alpha_{m}}\left(\mathbb{R}^{n}\right)\right\}
$$

and is equipped with the (quasi-)norm

$$
\|u\|_{\sigma ; p, q ; \alpha_{1}, \ldots, \alpha_{m}}:=\|f\|_{p, q ; \alpha_{1}, \ldots, \alpha_{m}} .
$$

(By $f * g$ we mean the convolution of functions $f$ and $g$.)

Let $\mathcal{L}$ be the class of all continuous functions $\lambda:(0, \infty) \rightarrow(0, \infty)$ which are increasing on some interval $(0, \delta)$, with $\delta=\delta(\lambda)>0$, and satisfy $\lim _{\lambda \rightarrow 0_{+}} \lambda(t)=0$. Let $\lambda \in \mathcal{L}$ and let $\Omega$ be a domain in $\mathbb{R}^{n}$. The space $C^{0, \lambda(\cdot)}(\bar{\Omega})$ of $\lambda(\cdot)$-Höldercontinuous functions consists of all those functions $u \in C(\bar{\Omega})$ for which the norm

$$
\|u\|_{C^{0, \lambda(\cdot)}(\bar{\Omega})}:=\sup _{x \in \Omega}|u(x)|+\sup _{\substack{x, y \in \Omega \\ x \neq y}} \frac{|u(x)-u(y)|}{\lambda(|x-y|)}
$$


is finite. Here $C(\bar{\Omega})$ stands for the family of all functions which are bounded and uniformly continuous on $\Omega$. (For more information about such spaces see [1] or [10].)

We write $A \lesssim B$ (or $A \gtrsim B$ ) if $A \leq c B$ (or $c A \geq B$ ) for some positive constant $c$ independent of appropriate quantities involved in the expressions $A$ and $B$, and $A \approx B$ if $A \lesssim B$ and $A \gtrsim B$. If $p \in[1, \infty]$, the conjugate number $p^{\prime}$ is defined by $\frac{1}{p}+\frac{1}{p^{\prime}}=1$ with the understanding that $1^{\prime}=\infty$ and $\infty^{\prime}=1$.

In [8] we have extended Theorem 4.9 of [5] (to the range $\sigma \in(0,1)$ ) and proved the following embedding.

Theorem 1. Let $0<\sigma<1, \frac{n}{\sigma}<p<\infty, 1<q<\infty, m \in \mathbb{N}, \alpha_{1}, \ldots, \alpha_{m} \in \mathbb{R}$ and let

$$
\lambda(t)=t^{\sigma-\frac{n}{p}} \prod_{j=1}^{m} \ell_{j}^{-\alpha_{j}}(t), \quad t>0 .
$$

Then

$$
H^{\sigma} L_{p, q ; \alpha_{1}, \ldots, \alpha_{m}}\left(\mathbb{R}^{n}\right) \hookrightarrow C^{0, \lambda(\cdot)}\left(\overline{\mathbb{R}^{n}}\right) .
$$

The aim of this paper is to show that the embedding of $H^{\sigma} L_{p, q ; \alpha_{1}, \ldots, \alpha_{m}}\left(\mathbb{R}^{n}\right)$ into $C^{0, \lambda(\cdot)}(\bar{\Omega})$, where $\Omega$ is a nonempty domain in $\mathbb{R}^{n}$, cannot be compact and that the embedding (2) is sharp with respect to the function $\lambda$.

\section{Main result and proofs}

Our main result reads as follows.

Theorem 2. Let the assumptions of Theorem 1 be satisfied. Let $n \geq 2$ and $\Omega \subseteq \mathbb{R}^{n}$ be a nonempty domain. Then the embedding

$$
H^{\sigma} L_{p, q ; \alpha_{1}, \ldots, \alpha_{m}}\left(\mathbb{R}^{n}\right) \hookrightarrow C^{0, \lambda(\cdot)}(\bar{\Omega})
$$

is not compact. Moreover, if a function $\mu \in \mathcal{L}$ satisfies $\frac{\mu}{\lambda} \in \mathcal{L}$, then the embedding

$$
H^{\sigma} L_{p, q ; \alpha_{1}, \ldots, \alpha_{m}}\left(\mathbb{R}^{n}\right) \hookrightarrow C^{0, \mu(\cdot)}(\bar{\Omega})
$$

does not hold.

To prove Theorem 2, we need some preliminary work. We modify the idea from [7] to construct suitable test functions. Assume that $\mathcal{G}$ is a function with the following properties:

$\mathcal{G}$ is positive and continuous on $(0,1]$;

$t \mathcal{G}(t)$ is nonincreasing on $\left(0, r_{0}\right]$, where $r_{0} \in(0,1]$ is a fixed number;

$\mathcal{G}\left(\frac{t}{2}\right) \lesssim \mathcal{G}(t), t \in(0,1]$ 
(notice that the assumption (5) is stronger than (4.2) of [7]). We use mollifiers to assign to the function $\mathcal{G}$ a family of functions $\left\{\mathcal{G}_{r}\right\}$. Let $\varphi \in C_{0}^{\infty}(\mathbb{R})$ be a non-negative function such that $\int_{\mathbb{R}} \varphi=1$ and $\operatorname{supp} \varphi=[-1,1]$. We define the function $\varphi_{\varepsilon}, \varepsilon>0$, by

$$
\varphi_{\varepsilon}(t):=\frac{1}{\varepsilon} \varphi\left(\frac{t}{\varepsilon}\right), \quad t \in \mathbb{R}
$$

and we put

$$
\psi:=\chi_{\left[-2+\frac{1}{16}, \frac{3}{4}-\frac{1}{16}\right]} * \varphi_{\frac{1}{16}} .
$$

Now, we extend $\mathcal{G}$ by zero outside the interval $(0,1]$ and we define functions $\mathcal{G}_{r}$, $r \in(0,1)$, by

$$
\mathcal{G}_{r}(t):=\left(\left(\chi_{[r, \infty)} \psi \mathcal{G}\right) * \varphi_{\frac{r}{4}}\right)(t), \quad t \in \mathbb{R} .
$$

For any $r \in\left(0, \frac{1}{4}\right)$, let $a_{r}$ be a positive number, let

$$
h_{r}(x):=a_{r} \mathcal{G}_{r}(|x|), \quad x \in \mathbb{R}^{n},
$$

and

$$
u_{r}(x):=x_{1}\left(g_{\sigma} * h_{r}\right)(x), \quad x=\left(x_{1}, \ldots, x_{n}\right) \in \mathbb{R}^{n} .
$$

Our first aim is to show that the functions $u_{r}$ belong to the source space in (3). To this end, we shall need the following result.

Lemma 1 (cf. Lemma 4.1 of [7]). Let $r \in\left(0, \frac{1}{4}\right)$ and let $\mathcal{G}_{r}$ be the functions defined by (7), where $\mathcal{G}$ satisfies (4)-(6). Then

$$
\mathcal{G}_{r} \in C_{0}^{\infty}(\mathbb{R}), \quad \operatorname{supp} \mathcal{G}_{r} \subset\left[\frac{r}{2}, 1\right] \quad \text { and } \quad \mathcal{G}_{r} \geq 0 .
$$

Moreover, there are positive constants $C_{1}$ and $C_{2}$ (independent of $r$ and $t$ ) such that

$$
\begin{array}{ll}
\mathcal{G}_{r}(t) \leq C_{1} \mathcal{G}(t) \chi_{\left[\frac{r}{2}, 1\right]}(t), & t \in(0,1] \\
\mathcal{G}_{r}(t) \geq C_{2} \mathcal{G}(t), & t \in\left[2 r, \frac{1}{2}\right] .
\end{array}
$$

We shall make use of the next assertions.

Lemma 2. Let $h$ belong to the Schwartz space $\mathcal{S}, \sigma \geq 0, j \in\{1, \ldots, n\}$ and let $\mathcal{R}_{j}$ be the Riesz transform. Then there exists a finite measure $\nu$ on $\mathbb{R}^{n}$ such that, for any $x=\left(x_{1}, \ldots, x_{n}\right) \in \mathbb{R}^{n}$,

$$
x_{j}\left(g_{\sigma} * h\right)(x)=-\sigma(2 \pi)^{-\frac{n}{2}}\left[g_{\sigma} *\left(\mathcal{R}_{j}\left(\nu * g_{1} * h\right)\right)\right](x)+\left[g_{\sigma} *\left(y_{j} h(y)\right)\right](x) .
$$

Proof. The equality can be derived analogously to (4.48) in [7]. 
Lemma 3 (cf. Cor. 4.12 of [7]). Let $1<p<\infty, 1 \leq q \leq \infty, \alpha_{1}, \ldots, \alpha_{m} \in \mathbb{R}$ and let $\nu$ be the measure from Lemma 2. Then, for all $f \in L_{p, q ; \alpha_{1}, \ldots, \alpha_{m}}\left(\mathbb{R}^{n}\right)$,

$$
\begin{aligned}
\left\|g_{\alpha} * f\right\|_{p, q ; \alpha_{1}, \ldots, \alpha_{m}} & \lesssim\|f\|_{p, q ; \alpha_{1}, \ldots, \alpha_{m}}, \quad \alpha \geq 0, \\
\left\|\mathcal{R}_{j} f\right\|_{p, q ; \alpha_{1}, \ldots, \alpha_{m}} & \lesssim\|f\|_{p, q ; \alpha_{1}, \ldots, \alpha_{m}}, \quad j=1, \ldots, n \\
\|\nu * f\|_{p, q ; \alpha_{1}, \ldots, \alpha_{m}} & \lesssim\|f\|_{p, q ; \alpha_{1}, \ldots, \alpha_{m}}
\end{aligned}
$$

We shall also need the following estimate.

Lemma 4. Let $n \geq 2, p>\frac{n}{n-1}, q \in[1, \infty], \frac{1}{p}=\frac{1}{\widetilde{p}}-\frac{1}{n}, \alpha_{1}, \ldots, \alpha_{m} \in \mathbb{R}$. Then, for all $f \in L_{\widetilde{p}, q ; \alpha_{1}, \ldots, \alpha_{m}}\left(\mathbb{R}^{n}\right)$,

$$
\left\|g_{1} * f\right\|_{p, q ; \alpha_{1}, \ldots, \alpha_{m}} \lesssim\|f\|_{\widetilde{p}, q ; \alpha_{1}, \ldots, \alpha_{m}} .
$$

Proof. The assumption $p>\frac{n}{n-1}$ and the equality $\frac{1}{p}=\frac{1}{\widetilde{p}}-\frac{1}{n}$ imply that $\widetilde{p} \in(1, n)$. Thus, the result follows on applying Theorem 3.1 of [7].

Lemma 5. Let $p, q \in(1, \infty), \alpha_{1}, \ldots, \alpha_{m} \in \mathbb{R}$. Let $g$ be a positive function which is continuous in $(0,1]$ and nonincreasing in some interval $\left(0, r_{0}\right] \subset(0,1]$. Then, for all $r \in\left(0, r_{0}\right)$,

$$
\left\|g(|y|) \chi_{[r, 1]}(|y|)\right\|_{p, q ; \alpha_{1}, \ldots, \alpha_{m}} \lesssim \mathcal{V}_{1}(r)+\mathcal{V}_{2}(r)
$$

where

$$
\begin{aligned}
& \mathcal{V}_{1}(r):=\left\|t^{\frac{n}{p}-\frac{1}{q}}\left(\prod_{j=1}^{m} \ell_{j}^{\alpha_{j}}(t)\right) g(t)\right\|_{q ;(r, 1)} \\
& \mathcal{V}_{2}(r):=r^{\frac{n}{p}}\left(\prod_{j=1}^{m} \ell_{j}^{\alpha_{j}}(r)\right) g(r) .
\end{aligned}
$$

Proof. The estimate can be proved analogously to the estimate (4.3) in Lemma 4.1 of [4].

The next lemma provides the upper estimate of $\left\|u_{r}\right\|_{\sigma ; p, q ; \alpha_{1}, \ldots, \alpha_{m}}$, where $u_{r}$ are the functions given by (9).

Lemma 6. Let $n \geq 2, p>\frac{n}{n-1}, q \in(1, \infty), \alpha_{1}, \ldots, \alpha_{m} \in \mathbb{R}$. Then the functions $u_{r}, r \in\left(0, r_{0}\right)$, defined by (9) (with $\mathcal{G}$ given by (4)-(6)), satisfy

$$
\left\|u_{r}\right\|_{\sigma ; p, q ; \alpha_{1}, \ldots, \alpha_{m}} \lesssim a_{r}\left(\mathcal{W}_{1}(r / 2)+\mathcal{W}_{2}(r / 2)\right),
$$

where

$$
\begin{aligned}
& \mathcal{W}_{1}(r):=\left\|t^{\frac{n}{p}+1-\frac{1}{q}}\left(\prod_{j=1}^{m} \ell_{j}^{\alpha_{j}}(t)\right) \mathcal{G}(t)\right\|_{q ;(r, 1)} \\
& \mathcal{W}_{2}(r):=r^{\frac{n}{p}+1}\left(\prod_{j=1}^{m} \ell_{j}^{\alpha_{j}}(r)\right) \mathcal{G}(r) .
\end{aligned}
$$


Proof. Since $u_{r} \in \mathcal{S}$ (cf. (10) and the fact that $g_{\sigma} * f \in \mathcal{S}$ for $f \in \mathcal{S}$ and $\sigma>0$ ), we can use Lemma 2 and the definition in (1) to get

$$
\begin{aligned}
& \left\|u_{r}\right\|_{\sigma ; p, q ; \alpha_{1}, \ldots, \alpha_{m}} \\
& \quad \lesssim\left\|g_{\sigma} * \mathcal{R}_{1}\left(\nu * g_{1} * h_{r}\right)\right\|_{\sigma ; p, q ; \alpha_{1}, \ldots, \alpha_{m}}+\left\|g_{\sigma} *\left(y_{1} h_{r}(y)\right)\right\|_{\sigma ; p, q ; \alpha_{1}, \ldots, \alpha_{m}} \\
& \quad=\left\|\mathcal{R}_{1}\left(\nu * g_{1} * h_{r}\right)\right\|_{p, q ; \alpha_{1}, \ldots, \alpha_{m}}+\left\|y_{1} h_{r}(y)\right\|_{p, q ; \alpha_{1}, \ldots, \alpha_{m}} .
\end{aligned}
$$

Applying Lemma 3, Lemma 4, (8) and (11) to the first term, we obtain

$$
\begin{aligned}
\left\|\mathcal{R}_{1}\left(\nu * g_{1} * h_{r}\right)\right\|_{p, q ; \alpha_{1}, \ldots, \alpha_{m}} & \lesssim\left\|g_{1} * h_{r}\right\|_{p, q ; \alpha_{1}, \ldots, \alpha_{m}} \\
& \lesssim\left\|h_{r}\right\|_{\tilde{p}, q ; \alpha_{1}, \ldots, \alpha_{m}} \\
& \lesssim a_{r}\left\|\mathcal{G}(|y|) \chi_{\left[\frac{r}{2}, 1\right]}(|y|)\right\|_{\tilde{p}, q ; \alpha_{1}, \ldots, \alpha_{m}} .
\end{aligned}
$$

Moreover, using Lemma 5 with $g=\mathcal{G}$ (observe that this function satisfies the assumptions of Lemma 5) and the identity $\frac{n}{\widetilde{p}}=\frac{n}{p}+1$, we arrive at

$$
\left\|\mathcal{G}(|y|) \chi_{\left[\frac{r}{2}, 1\right]}(|y|)\right\|_{\widetilde{p}, q ; \alpha_{1}, \ldots, \alpha_{m}} \lesssim \mathcal{W}_{1}(r / 2)+\mathcal{W}_{2}(r / 2) .
$$

Consequently,

$$
\left\|\mathcal{R}_{1}\left(\nu * g_{1} * h_{r}\right)\right\|_{p, q ; \alpha_{1}, \ldots, \alpha_{m}} \lesssim a_{r}\left[\mathcal{W}_{1}(r / 2)+\mathcal{W}_{2}(r / 2)\right]
$$

Furthermore, we use (8), (11) and Lemma 5 with $g(t)=t \mathcal{G}(t)$ to get

$$
\begin{aligned}
\left\|y_{1} h_{r}(y)\right\|_{p, q ; \alpha_{1}, \ldots, \alpha_{m}} & \leq\left\||y| h_{r}(y)\right\|_{p, q ; \alpha_{1}, \ldots, \alpha_{m}} \\
& \lesssim a_{r}\left\||y| \mathcal{G}(|y|) \chi_{\left[\frac{r}{2}, 1\right]}(|y|)\right\|_{p, q ; \alpha_{1}, \ldots, \alpha_{m}} \\
& \lesssim a_{r}\left[\mathcal{W}_{1}(r / 2)+\mathcal{W}_{2}(r / 2)\right] .
\end{aligned}
$$

Finally, by (12), (13) and (14) we obtain the result.

To prove the non-compactness of the embedding (3), we shall need the following assertion.

Lemma 7. Let $\sigma \in(0, n), R \in\left(0, \frac{1}{4}\right)$ and let

$$
a_{r} \leq C \text { for all } r \in\left(0, \frac{1}{4}\right) \text { with some } C \in(0, \infty) \text {. }
$$

Moreover, let the function $\mathcal{G}$ from (4)-(6) and the numbers $a_{r}$ satisfy

$$
a_{r} \int_{2 r}^{\frac{R}{2}} t^{\sigma-1} \mathcal{G}(t) \mathrm{d} t \rightarrow \infty \quad \text { as } \quad r \rightarrow 0_{+} .
$$

Then there exist $\varepsilon=\varepsilon(\sigma) \in\left(0, \frac{1}{2}\right), r_{1}=r_{1}(R) \in\left(0, \frac{R}{4}\right)$ and a positive constant $c$ (independent of $R$ and $r_{1}$ ) such that for the functions $u_{r}$ defined by (9), (8) and (7),

$$
\left|\left[u_{r}(x)-u_{R}(x)\right]-\left[u_{r}(0)-u_{R}(0)\right]\right| \geq \operatorname{cr} a_{r} \int_{2 r}^{\frac{R}{2}} t^{\sigma-1} \mathcal{G}(t) \mathrm{d} t
$$

for every $r \in\left(0, r_{1}\right)$ and $x=(\varepsilon r, 0, \ldots, 0) \in \mathbb{R}^{n}$. 
Proof. The result immediately follows from Lemma 4.5 of [7].

Now, we are ready to prove the main result.

Proof of Theorem 2. We can suppose without loss of generality that

$$
B:=\left\{x \in \mathbb{R}^{n} ;|x| \leq 1\right\} \subset \Omega .
$$

Let $r \in\left(0, \frac{1}{4}\right)$. Take $\gamma<0$ and put

$$
\mathcal{G}(t)=t^{\gamma-1-\frac{n}{p}} \prod_{j=1}^{m} \ell_{j}^{-\alpha_{j}}(t), \quad t \in(0,1], \quad \text { and } \quad a_{r}=r^{-\gamma} .
$$

The function $\mathcal{G}$ satisfies (4)-(6). Thus, by Lemma 6 ,

$$
\left\|u_{r}\right\|_{\sigma ; p, q ; \alpha_{1}, \ldots, \alpha_{m}} \lesssim r^{-\gamma}\left[\left(\int_{\frac{r}{2}}^{1} t^{\gamma q} \frac{\mathrm{d} t}{t}\right)^{\frac{1}{q}}+r^{\gamma}\right] \lesssim 1 \quad \text { for all } r \in\left(0, r_{0}\right),
$$

where $u_{r}$ are the functions given by (9). (Observe, that the assumptions $\sigma \in(0,1)$ and $n \geq 2$ yield $p>\frac{n}{\sigma}>n>\frac{n}{n-1}$.)

Taking $R \in\left(0, \frac{1}{4}\right)$, we can see that the conditions (15) and (16) are satisfied and so, by Lemma 7 , there exists $\varepsilon \in\left(0, \frac{1}{2}\right)$ and $r_{1} \in\left(0, \frac{R}{4}\right)$ and a positive constant $c$ (independent of $R$ and $r_{1}$ ) such that

$$
\begin{aligned}
& \left|\left[u_{r}(x)-u_{R}(x)\right]-\left[u_{r}(0)-u_{R}(0)\right]\right| \\
& \quad \geq c r^{1-\gamma} \int_{2 r}^{\frac{R}{2}} t^{\sigma-1+\gamma-1-\frac{n}{p}} \prod_{j=1}^{m} \ell_{j}^{-\alpha_{j}}(t) \mathrm{d} t \approx r^{\sigma-\frac{n}{p}} \prod_{j=1}^{m} \ell_{j}^{-\alpha_{j}}(r)=\lambda(r)
\end{aligned}
$$

for every $r \in\left(0, r_{1}\right)$ and $x=(\varepsilon r, 0, \ldots, 0)$. Consequently, for any fixed $R \in\left(0, \frac{1}{4}\right)$ and every sufficiently small positive $r$,

$$
\left\|u_{r}-u_{R}\right\|_{C^{0, \lambda(\cdot)(\bar{\Omega})}} \geq \frac{\left|\left[u_{r}(x)-u_{R}(x)\right]-\left[u_{r}(0)-u_{R}(0)\right]\right|}{\lambda(\varepsilon r)} \geq c \frac{\lambda(r)}{\lambda(\varepsilon r)} \geq c_{0},
$$

where $c$ and $c_{0}$ are positive constants independent of $R$ and $r$.

Finally, consider the sequence of functions $\left\{u_{1 / k}\right\}_{k=k_{0}}^{\infty}$ with $k_{0}$ sufficiently large. By (19), this sequence is bounded in $H^{\sigma} L_{p, q ; \alpha_{1}, \ldots, \alpha_{m}}\left(\mathbb{R}^{n}\right)$ however, in view of $(20)$, it has no Cauchy subsequence in $C^{0, \lambda(\cdot)}(\bar{\Omega})$. Therefore, the embedding (3) is not compact.

To prove sharpness, suppose that there is a function $\mu \in \mathcal{L}$ such that $\frac{\mu}{\lambda} \in \mathcal{L}$ and $H^{\sigma} L_{p, q ; \alpha_{1}, \ldots, \alpha_{m}}\left(\mathbb{R}^{n}\right) \hookrightarrow C^{0, \mu(\cdot)}(\bar{\Omega})$ for some nonempty domain $\Omega$ in $\mathbb{R}^{n}$. Take a ball $B \subset \Omega$. Then

$$
H^{\sigma} L_{p, q ; \alpha_{1}, \ldots, \alpha_{m}}\left(\mathbb{R}^{n}\right) \hookrightarrow C^{0, \mu(\cdot)}(\bar{B})
$$


Moreover, by Lemma 4.15 (iv) of [5], the condition $\frac{\mu}{\lambda} \in \mathcal{L}$ implies that

$$
C^{0, \mu(\cdot)}(\bar{B}) \hookrightarrow \hookrightarrow C^{0, \lambda(\cdot)}(\bar{B}) .
$$

Combining this embedding with (21), we arrive at

$$
H^{\sigma} L_{p, q ; \alpha_{1}, \ldots, \alpha_{m}}\left(\mathbb{R}^{n}\right) \hookrightarrow \hookrightarrow C^{0, \lambda(\cdot)}(\bar{B}),
$$

which contradict the non-compactness of the embedding (3) with $\Omega=B$.

\section{References}

[1] Adams, R. A.: Sobolev spaces. New York: Academic Press 1975.

[2] Edmunds, D. E., Gurka, P. and Opic, B., Double exponential integrability of convolution operators in generalized Lorentz-Zygmund spaces. Indiana Univ. Math. J. 44 (1995), 19 - 43.

[3] Edmunds, D. E., Gurka, P. and Opic, B., Double exponential integrability, Bessel potentials and embedding theorems. Studia Math. 115 (1995), 151 - 181.

[4] Edmunds, D. E., Gurka, P. and Opic, B., Sharpness of embeddings in logarithmic Bessel-potential spaces. Proc. Roy. Soc. Edinburgh 126A (1996), 995 - 1009.

[5] Edmunds, D. E., Gurka, P. and Opic, B., On embeddings of logarithmic Bessel potential spaces. J. Funct. Anal. 146 (1997), $116-150$.

[6] Edmunds, D. E., Gurka, P. and Opic, B., Norms of embeddings of logarithmic Bessel potential spaces. Proc. Amer. Math. Soc. 126 (1998), $2417-2425$.

[7] Edmunds, D. E., Gurka, P. and Opic, B., Optimality of embeddings of logarithmic Bessel potential spaces. Quart. J. Math. Oxford Ser. (2) 51 (2000), $185-209$.

[8] Edmunds, D. E., Gurka, P. and Opic, B., Compact and continuous embeddings of logarithmic Bessel potential spaces. Studia Math. 168 (2005), 229 - 250.

[9] Evans, W. D., Opic, B. and Pick, L., Interpolation of operators on scales of generalized Lorentz-Zygmund spaces. Math. Nachr. 182 (1996), 127 - 181.

[10] Kufner, A., John, O. and Fučík, S., Function spaces. Prague: Academia 1977.

[11] Opic, B. and Pick, L., On generalized Lorentz-Zygmund spaces. Math. Inequal. Appl. 2 (1999), $391-467$.

Received August 9, 2004 